\title{
Decreased Hepatic Phosphatidylinositol- 3,4,5-Triphosphate (PIP3) Levels and Impaired Glucose Homeostasis in Type 1 and Type 2 Diabetic Rats
}

\author{
Prasenjit Manna Sushil K. Jain \\ Department of Pediatrics, Louisiana State University Health Sciences Center, Shreveport, LA
}

\section{Key Words}

PIP3 • PIP2 • Type 1 and Type 2 Diabetic Rats • Livers • Glucose metabolism

\begin{abstract}
Background/Aims: Phosphatidylinositol-3,4,5-triphosphate (PIP3) and phosphatidylinositol4,5-biphosphate (PIP2) are two well-known lipid second messengers. Polyphosphoinositides have been implicated in the regulation of the signal transduction pathways involved in glucose metabolism using cell culture studies. However, there are no in vivo studies in the literature investigating the status of PIP3 and PIP2 in any of the tissues of diabetic animals. The liver plays an important role in the regulation of whole body glucose homeostasis. This study investigated whether hepatic PIP3 and/or PIP2 levels are altered in diabetes. Methods: Experiments were performed in streptozotocin-treated type 1 (T1D) and ZDF type 2 (T2D) diabetic rats. Blood glucose was determined utilizing glucose oxidase, glycosylated hemoglobin (GHb) using Glyco-Tek Affinity columns, and hepatic PIP3 and PIP2 concentrations by sandwich ELISA, and Akt phosphorylation and GLUT2 protein abundance by Western blotting. Results: Blood glucose and GHb were higher in T1D and T2D rats compared to controls. As compared to control animals, in livers from T1D and T2D rats PIP3 levels were reduced, AKT phosphorylation downregulated, and GLUT2 protein expression increased. PIP2 levels were unchanged. Conclusion: PIP3 is decreased, AKT phosphorylation downregulated, GLUT2 protein expression increased and glucose homeostasis altered in livers of type 1 and type 2 diabetic rats.
\end{abstract}

Copyright $(2012$ S. Karger AG, Basel

\section{Introduction}

Polyphosphoinositides (PIs) play an essential role in diverse cellular functions depending upon the phosphorylation status of their inositol group [1]. PIP3 (phosphatidylinositol- 
3,4,5-triphosphate) and PIP2 (phosphatidylinositol-4,5-biphosphate) are two well known signaling molecules [2]. PIP3 controls a complex intracellular signaling network that regulates many cellular processes, such as cell growth, proliferation, and survival [3]. PIP3 target proteins are located in the cytosol of unstimulated cells and are activated via binding of the PH (pleckstrin homology) domain with PIP3 [4]. This lipid molecule regulates a variety of PH domain-containing proteins, such as serine-threonine kinase AKT and PDK1, GRP1, a GDP/GTP exchange factor of ADP ribosylating factor 6 , and protein tyrosine kinases of the Bruton's tyrosine kinase (Btk) and Tec families [5, 6]. This diversity in PIP3 signaling makes this molecule an important lipid second messenger downstream from growth factor and oncogene signaling cascades. On the other hand, PIP2 regulates a variety of diverse cellular activities, including modulation of the actin cytoskeleton, endocytosis, exocytosis, and ion channel activity [7].

Insulin stimulated glucose uptake and metabolism is one of the fundamental mechanisms responsible for the maintenance of glucose homeostasis in the body. Impaired insulin action or insulin resistance leads to type 2 diabetes (T2D). PI3K (phosphoinositide3-kinase) and PTEN (phosphatase and tensin homologue deleted on chromosome 10) play central roles in the insulin signaling cascade and glucose metabolism [8]. The activation of Class I PI3K causes phosphorylation of PIP2 at position 3 of its inositol head group, leading to the formation of PIP3, but PTEN activation causes the degradation of PIP3 [1]. This means that the cellular PIP3 concentration is regulated by the PI3K/PTEN equilibrium. Studies in the literature have shown that inhibition of PTEN expression using PTEN antisense oligonucleotides normalized blood glucose levels in ob/ob (obese) mice [9] and that overexpression of PTEN resulted in inhibition of PIP3 production and glucose uptake [10]. Similarly, various studies using knockout mice and cell models of PTEN and PI3K have documented that inhibition of PTEN, and activation of PI3K and AKT (serine/ threonine protein kinase) signaling molecules, are crucial in insulin signaling pathways and the maintenance of whole body glucose metabolism [11]. These findings suggest that PIP3 can play a role in the insulin signaling pathway [1,3]. Our previous study in adipocyte (3T3L1) cell model indicates that high glucose exposure caused a decrease in cellular PIP3, downregulation of AKT phosphorylation and GLUT4 (glucose transporter 4) expression, and reduction of glucose utilization [12]. Exogenous PIP3 supplementation however could upregulate the AKT phosphorylation and GLUT4 expression, and glucose utilization in high glucose treated adipocytes. Using 3T3L1 adipocytes, Funaki et al. [13] also reported that in the presence of a cell permeable phosphoinositide-binding peptide (PBP10), PIP2 functions as a second messenger in GLUT4 activation and glucose uptake, possibly through regulation of F-actin remodeling. However, there are no in vivo studies in the literature investigating the status of PIP3 and PIP2 in any of the tissues of diabetic animals. The liver plays a crucial role in the maintenance of body glucose homeostasis, and hepatic insulin resistance is a major risk factor in the development of diabetes and its associated complications. Using both type 1 (TID) and type 2 diabetic (T2D) rats, this study investigated the status of PIP3 and PIP2 in the liver tissues of diabetic animals and their effect on hepatic glucose metabolism. Livers from both T1D and T2D rats showed a reduction in hepatic PIP3 levels, downregulation of AKT phosphorylation, and an increase in GLUT2 (glucose transporter 2) protein expression; however, PIP2 levels were unchanged. This study demonstrates for the first time a decrease in hepatic levels of PIP3 but not those of PIP2, which may have a role in the impaired glucose homeostasis in type 1 and type 2 diabetic rats.

\section{Materials and Methods}

\section{Materials}

GLUT2 antibody was purchased from Millipore (Billerica, MA). AKT and phospho AKT (serine 473) were purchased from Cell Signaling Technology (Beverly, MA). All other chemicals were purchased from Sigma Chemical Co. (St. Louis, MO) unless otherwise mentioned. 


\section{Cellular Physiology Cell Physiol Biochem 2012;30:1363-1370 \begin{tabular}{l|l} 
DOI: 10.1159/000343325 & (C) 2012 S. Karger AG, Basel
\end{tabular} and Biochemistry Published online: October 30, 2012 www.karger.com/cpb \\ Manna/Jain: PIP3, PIP2 and Hepatic Glucose Homeostasis}

\section{Animal studies}

All procedures used in our study were in accordance with the ethical standards of the institution, and prior approval was obtained from the institutional Animal Welfare Committee.

\section{Studies with type 1 diabetic animals}

Male Sprague Dawley (SD) rats were purchased at 5 weeks of age (200-220 g) from Charles River (Wilmington, MA) and allowed 2 days for environmental and trainer handling acclimation. The rats were fasted overnight and then injected with streptozotocin (STZ) intraperitoneally at a dose of $65 \mathrm{mg} / \mathrm{kg}$ body weight in citrate buffer ( $\mathrm{pH} 4.5)$. Control rats were injected with citrate buffer alone to serve as a normal control group (Cont) for type 1 diabetes (T1D). The rats were tested for hyperglycemia (blood glucose $>300$ $\mathrm{mg} / \mathrm{dL}$ ) by measuring their blood glucose concentrations at 3 and 7 days after the STZ injections. The rats were maintained under standard housing conditions at $22 \pm 2^{\circ} \mathrm{C}$ with $12 / 12$-h light/dark cycles and fed with a standard 8640 lab chow diet (Harlan, Indianapolis, IN). At the age of 14 weeks, the rats were fasted overnight and then euthanized by exposure to isoflurane (Webster Veterinary Supply Inc., Devens, MA) on the next day for analysis.

\section{Studies with type 2 diabetic animals}

Male Zucker Diabetic Fatty (ZDF) rats were purchased at 5 weeks of age (200-220 g) from Charles River (Wilmington, MA) and allowed 2 days for environmental and trainer handling acclimation. Rats were fasted overnight and then weighed. The rats were maintained under standard housing conditions at $22 \pm$ $2{ }^{\circ} \mathrm{C}$ with 12/12-h light/dark cycles and fed with a high-calorie Purina 5008 lab chow diet (Charles River). The rats were tested for hyperglycemia by measuring their blood glucose concentrations. At 14 weeks of age the ZDF rats became diabetic (blood glucose $>300 \mathrm{mg} / \mathrm{dL}$ ) (T2D). For age matched controls, male Sprague Dawley (SD) rats were also purchased (Charles River) at the age of $5 \mathrm{wks}$, fed a normal diet, and maintained under similar conditions until they reached 14 weeks of age. In addition, normoglycemic prediabetic ZDF rats ( $6 \mathrm{wks}$ ) were used as baseline (BL) controls. At the end of the experiment, diabetic ZDF rats (T2D), nondiabetic SD rats (age-matched controls) and baseline ZDF rats (BL) were fasted overnight and euthanized by exposure to isoflurane (Webster Veterinary Supply Inc., Devens, MA) on the next day for analysis.

For the assessment of blood glucose levels, blood was obtained via tail incision and measured using an Advantage Accu-Chek glucometer (Boehringer Mannheim Corp., Indianapolis, IN). Blood was collected via heart puncture with a $19^{1 / 2}$-gauge needle into EDTA Vacutainer tubes. Plasma was isolated after centrifuging the blood in a $4^{\circ} \mathrm{C}$ centrifuge at $1500 \mathrm{rpm}$ for $10 \mathrm{~min}$.

Measurement of blood glucose, glycosylated hemoglobin (GHb), and liver function tests

Blood glucose levels were determined by measuring glucose oxidase using an Accu-Chek Advantage glucometer (Boehringer Mannheim). GHb was determined using Glyco-Tek Affinity column kits and reagents (Cat. No. 5351) purchased from Helena Laboratories (Beaumont, TX, USA). A portion of blood from rats in each group was sent to the clinical laboratory of LSUHSC-Shreveport (located in the same building) for clinical tests to determine liver function.

\section{Preparation of liver tissue homogenates}

Liver tissues excised from the experimental rats were immediately perfused with cold saline to remove left over blood, immediately frozen using liquid nitrogen, then ground well into powders and stored at $-70^{\circ} \mathrm{C}$ until further use. The frozen liver tissue $(\sim 150 \mathrm{mg})$ was washed by resuspending then in $1 \mathrm{~mL}$ PBS containing protease inhibitors, mildly vortexed, and centrifuged at $15,000 \mathrm{rpm}$ at $4^{\circ} \mathrm{C}$ for $10 \mathrm{~min}$. The supernatants were discarded and the cell pellets were washed once more as described above and then resuspended in $500 \mu \mathrm{L}$ radioimmuno precipitation assay (RIPA) buffer (50 mM Tris pH 8, $150 \mathrm{mM} \mathrm{NaCl}, 1 \%$ NP-40, 0.5\% deoxycholic acid, $0.1 \%$ SDS) supplemented with protease and phosphatase inhibitors (1 mM PMSF, $5 \mu \mathrm{g} /$ mL leupeptin, $2 \mu \mathrm{g} / \mathrm{mL}$ aprotinin, $1 \mathrm{mM}$ EDTA, $10 \mathrm{mM} \mathrm{NaF}$, and $1 \mathrm{mM} \mathrm{Na}_{3} \mathrm{VO}_{4}$ ), homogenized using a Dounce homogenizer, and subjected to mild sonication. The tubes were centrifuged at $15,000 \mathrm{rpm}\left(4^{\circ} \mathrm{C}, 30 \mathrm{~min}\right)$ and the supernatants (extracts) were collected. The collected extracts were subjected to centrifugation once more as described above and cell debris was removed. The protein content of the extracts was estimated using the BCA protein assay kit (Pierce/Thermo Scientific, Rockford, IL). 


\section{Cellular Physiology Cell Physiol Biochem 2012;30:1363-1370 \begin{tabular}{l|l|l} 
DOI: 10.1159/000343325 & (C) 2012 S. Karger AG, Basel
\end{tabular} \begin{tabular}{l|l|} 
and Biochemistry Published online: October 30, 2012 & www.karger.com/cpb \\
\cline { 1 - 2 }
\end{tabular}

\section{Measurement of PIP3 and PIP2 levels}

PIP3 and PIP2 concentrations in the liver tissue were measured by the sandwich ELISA method using commercially available kits from Echelon Biosciences, Inc. (Salt Lake City, UT). Before estimating the cellular PIP3 and PIP2 concentration, total phosphoinositides were extracted from the liver tissue homogenate following the method as described by Grey et al. [14] as well as in the kits. The frozen liver tissue $(\sim 150$ $\mathrm{mg}$ ) was homogenized in $50 \mathrm{mM}$ potassium phosphate buffer ( $\mathrm{pH}$ 6.9) containing $1 \mathrm{mM}$ EDTA and 1:100 $(\mathrm{v} / \mathrm{v})$ protease inhibitior cocktail (Calbiochem) followed by centrifugation at $15,000 \mathrm{Xg}$ for $30 \mathrm{~min}$ at $4^{\circ} \mathrm{C}$. The resulting supernatant was used to extract the phosphoinositides. Before extraction, proteins and the lipids present in the homogenates were precipitated by adding $0.5 \mathrm{~mL}$ ice cold $0.5 \mathrm{M}$ TCA. After standing on ice for $5 \mathrm{~min}$ the precipitate was pelleted by centrifugation at $1500 \mathrm{Xg}$ for $5 \mathrm{~min}$ at $4^{\circ} \mathrm{C}$. The pellet was then washed two times with $1 \mathrm{ml}$ of $5 \%$ TCA $1 \mathrm{mM}$ EDTA. Neutral lipids were extracted first from the pellet with methanol:chloroform $(2: 1)$ by vortexing three to four times over a period of $10 \mathrm{~min}$ at room temperature followed by centrifugation at $1500 \mathrm{Xg}$ for $5 \mathrm{~min}$ at $4^{\circ} \mathrm{C}$. This extraction was repeated and the supernatants were discarded. The acidic lipids were then extracted with methanol:chloroform: $12 \mathrm{M} \mathrm{HCl}$ (80:40:1) by vortexing occasionally over a $15 \mathrm{~min}$ period at room temperature followed by centrifugation at $1500 \mathrm{Xg}$ for $5 \mathrm{~min}$ at $4^{\circ} \mathrm{C}$. This extraction was repeated and the supernatant were collected. A phase split was then carried out by the addition of $750 \mu \mathrm{L}$ chloroform and $1.35 \mathrm{~mL} 0.1 \mathrm{M} \mathrm{HCl}$ followed by centrifugation to separate the organic and aqueous phases at $1500 \mathrm{X}$ g for $5 \mathrm{~min}$ at $4^{\circ} \mathrm{C}$. The organic phase was collected into a clean tube and dried under a stream of $\mathrm{N}_{2}$. The dried lipids were then resuspended in $60 \mu \mathrm{L}$ of the assay buffer (PBS-T 3\% Protein Stabilizer) provided in the manufacturer kit, vortexed and sonicated briefly in an ice water bath to dissolve the phosphoinositides. The mass of phosphoinositides were estimated using the respective ELISA kits (PIP3 Mass ELISA kit \# K-2500s and PIP2 Mass ELISA kit \# K-4500). Appropriate controls and standards (specified by each manufacturer's kit) were used every time.

\section{Immunoblotting}

All samples contained approximately the same amount of protein $(\sim 20-40 \mu \mathrm{g})$ and were run as $10 \%$ SDS-PAGE and transferred to a nitrocellulose membrane. Membranes were blocked at room temperature for $2 \mathrm{~h}$ in blocking buffer containing 1\% BSA to prevent non-specific binding and then incubated with: antiAKT (AKT2) (1:1000 dilution), anti-GLUT2 (1:1000), and anti-phosphorylated AKT (serine 473) (1:500) primary antibodies at $4{ }^{\circ} \mathrm{C}$ overnight. The membranes were washed in TBS-T $(50 \mathrm{mmol} / \mathrm{L}$ Tris- $\mathrm{HCl}, \mathrm{pH}$ 7.6, $150 \mathrm{mmol} / \mathrm{L} \mathrm{NaCl}, 0.1 \%$ Tween 20) for $30 \mathrm{~min}$ and incubated with the appropriate HRP conjugated secondary antibody (1:5000 dilution) for $2 \mathrm{~h}$ at room temperature and developed using the ultrasensitive ECL substrate (Millipore, MA). The intensity of each immunoblotting band was measured using the histogram tool of Adobe Photoshop CS5.

\section{Statistical analysis}

Data from cell culture studies were analyzed statistically using one way analysis of variance (ANOVA) with Sigma Stat statistical software (Jandel Scientific, San Rafael, CA). When data passed a normality test, all groups were compared using the Student-Newman-Keuls method. A difference was considered significant at the $\mathrm{p}<0.05$ level.

\section{Results}

Tables 1 and 2 show the levels of blood glucose, glycated hemoglobin (GHb), and the status of the serum enzymes related to hepatic dysfunction, ALT (alanine transaminase) and AP (alkaline phosphatase), in the blood samples of all the experimental animals. Results (Table 1) showed that STZ-treated T1D rats have higher levels of blood glucose and GHb compared to those seen in controls (Cont). In addition, T1D rats also have higher levels of ALT and AP compared to those of controls (Cont). Similarly, T2D rats also exhibit higher levels of blood glucose, GHb, and ALT and AP compared to those of age matched (14 wk) control SD rats and normoglycemic young (6 wk) ZDF rats (BL) (Table 2). We also observed that the body weights of T1D and T2D rats were reduced compared to those of age matched control rats. 


\section{Cellular Physiology Cell Physiol Biochem 2012;30:1363-1370}

\begin{tabular}{l|l} 
DOI: $10.1159 / 000343325$ & (c) 2012 S. Karger AG, Basel
\end{tabular} and Biochemistry Published online: October 30, 2012

\begin{tabular}{ccc}
\hline Parameters & Cont & T1D \\
\hline Age (wk) & 14 & 14 \\
Body weight (gm) & $376 \pm 6^{*}$ & $149 \pm 9^{\#}$ \\
Blood glucose (mg/dL) & $143 \pm 6.5^{*}$ & $446 \pm 24^{\#}$ \\
Blood GHb (\%) & $7.68 \pm 0.17^{*}$ & $16.98 \pm 0.38^{\#}$ \\
ALT (U/L) & $63.67 \pm 3.6^{*}$ & $178 \pm 36.2^{\#}$ \\
AP (U/L) & $14.8 \pm 2.75^{*}$ & $38.5 \pm 5.23^{\#}$
\end{tabular}

Each value represents the mean $\pm \operatorname{SE}(n=6)$. ALT, alanine aminotransferase; AP, alkaline phosphatase. Cont: SD rats at the age of $14 \mathrm{wks}$ and T1D: streptozotocin treated type 1 diabetic SD rats at the age of 14 wks. Differences between * vs \# are considered significant ( $\mathrm{p}<0.05)$.

Table 1. Age, body weight, blood glucose, glycosylated hemoglobin (GHb), and liver function tests (ALT and AP) of the experimental rats.

\begin{tabular}{|c|c|c|c|}
\hline Parameters & Cont & $\mathrm{T} 2 \mathrm{D}$ & BL \\
\hline Age (wk) & 14 & 14 & 6 \\
\hline Body weight (gm) & $439 \pm 18^{*}$ & $375 \pm 7 \#$ & $146 \pm 5^{* *}$ \\
\hline Blood glucose (mg/dL) & $115 \pm 16^{*}$ & $486 \pm 36^{\#}$ & $89 \pm 3.32^{*}$ \\
\hline Blood GHb (\%) & $5.39 \pm 0.14^{*}$ & $14.23 \pm 0.51^{\#}$ & $5.11 \pm 0.19^{*}$ \\
\hline $\operatorname{ALT}(\mathrm{U} / \mathrm{L})$ & $51.16 \pm 6.94^{*}$ & $128 \pm 12.8^{\#}$ & $65 \pm 3.53^{*}$ \\
\hline $\mathrm{AP}(\mathrm{U} / \mathrm{L})$ & $15.33 \pm 3.01^{*}$ & $49.5 \pm 7.67 \#$ & $9.2 \pm 1.36^{*}$ \\
\hline \multicolumn{4}{|c|}{$\begin{array}{l}\text { Each value represents the mean } \pm S E(n=6) \text {. ALT, alanine aminotransferase; AP, } \\
\text { alkaline phosphatase. Cont: SD rats at the age of } 14 \text { wks (age matched control); } \\
\text { T2D: type } 2 \text { diabetic ZDF rats at the age of } 14 \mathrm{wks} \text {; and BL: normoglycemic young } \\
\text { ZDF rats at the age of } 6 \text { wks. Differences between } * \text { vs \#, }{ }^{* *} \text { vs \# and }{ }^{*} \mathrm{vs}^{* *} \text { are } \\
\text { considered significant }(p<0.05) .\end{array}$} \\
\hline
\end{tabular}

Table 2. Age, body weight, blood glucose, glycosylated hemoglobin (GHb), and liver function tests (ALT and AP) of the experimental rats.

Figures 1 and 2 show the levels of PIP3, PIP2, and the expression of different signaling molecules (AKT and GLUT2) involved in glucose metabolism pathways in the liver tissues of normal and diabetic rats. The results in Figure 1 show that livers from T1D rats have lower levels of PIP3 (1C), along with downregulation of phospho AKT (1A) and upregulation of GLUT2 expression (1B), compared to that of controls. Livers from T2D rats also show lower PIP3 levels (2C), phosphorylation of AKT (2A), and an increase in GLUT2 (2B) protein expression compared to those seen in both age matched control SD rats and normoglycemic young ZDF rats (BL). However, PIP2 levels were unchanged in liver tissue of both types of rats compared to those seen in controls (1D and 2D). In the human body, the liver plays an important role in the regulation of glucose homeostasis, and an increased hepatic glucose output contributes to fasting hyperglycemia in diabetes [15]. The results from our in vivo studies demonstrate a reduction in hepatic PIP3 as well as impaired glucose metabolism in diabetic rats. 
Fig. 1. Levels of PIP3 and PIP2 and the expression of phospho AKT (serine 473)/ AKT (AKT2) and GLUT2 in the liver tissue of control and TID rats. Cont (control, 14 wk old SD rats), T1D (type 1 diabetic, STZ-treated $14 \mathrm{wk}$ old SD rats). A: phospho AKT/AKT; B: GLUT2; C: PIP3; and D: PIP2. Values are expressed as mean $\pm S E(n=6)$. Differences between * vs \# are considered significant ( $\mathrm{p}<0.05)$.

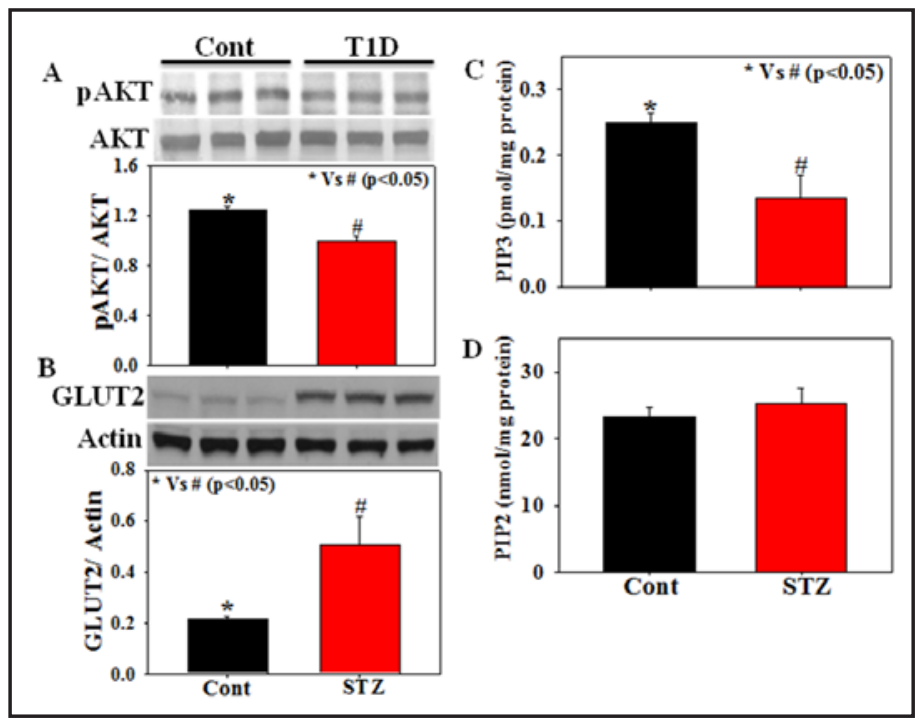

Fig. 2. Levels of PIP 3 and PIP2 and the expression of phospho AKT (serine 473)/ AKT (AKT2) and GLUT2 in the liver tissue of control and T2D rats. Cont (age matched control, 14 wk old SD rats), T2D (type 2 diabetic, 14 wk old ZDF rats) and BL (baseline, $6 \mathrm{wk}$ old normoglycemic ZDF rats). A: phospho AKT/AKT; B: GLUT2; C: PIP3; and D: PIP2. Values are expressed as mean \pm SE $(n=6)$. Differences between $*$ vs \#, ** vs \# and * vs ** are considered significant $(\mathrm{p}<0.05)$.

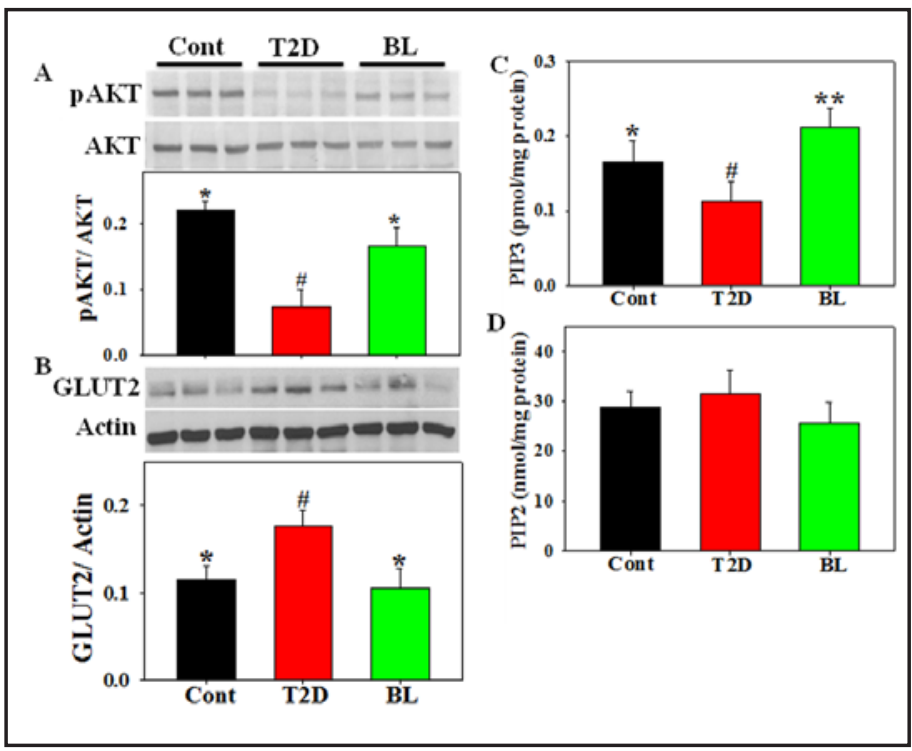

\section{Discussion}

The liver plays an important role in the regulation of body glucose homeostasis. Hepatic insulin resistance is one of the major risk factors in the pathogenesis of diabetes mellitus [15]. Polyphosphoinositides (PIs) play an essential role in diverse cellular functions depending upon the phosphorylation status of their inositol group [1]. Present study demonstrates for the first time that livers from T1D and T2D rats have lower PIP3 levels, downregulation of phospho AKT, and an increase in GLUT2 expression compared to those seen in controls, but that PIP2 levels were unchanged.

In our bodies glucose homeostasis is a tightly regulated phenomenon, which involves glucose uptake by peripheral tissues and glucose storage by, or production from the liver. Under normal conditions, the liver can store or release glucose as needed. In the postprandial state, glucose is taken up by the liver and is incorporated into glycogen and fatty acids or oxidized into $\mathrm{CO}_{2}$. However, in the fasting state, the liver releases glucose into the blood stream via glycogenolysis and gluconeogenesis. Thus glucose transport across the liver 
plasma membrane is a bidirectional process. In liver, GLUT2 is the main isoform of glucose transporters and due to its high $\mathrm{K}_{\mathrm{m}}$ value; it allows rapid equilibration of the intracellular with the extracellular glucose level within the physiological range of plasma glucose concentrations $[16,17]$. Studies in the literature suggest that under diabetic pathophysiology, hepatic glucose homeostasis is markedly disturbed due to increased hepatic insulin resistance [15, 18]. It has been reported that hepatic insulin resistance is associated with hepatic steatosis, causing impaired glycogen synthesis and increased gluconeogesis, which may cause altered glucose homeostasis in diabetic liver tissues $[15,19,20]$. Recent studies report that increased hepatic fat accumulation upregulates the concentration of hepatic diacylglycerol (DAG) [18]. The accumulation of DAG causes the activation of protein kinase C $\varepsilon$ (PKC $\varepsilon$ ), which subsequently inhibits insulin receptor kinase. This leads to decreased insulin stimulated tyrosine phosphorylation of insulin receptor substrate-1 and -2 (IRS-1, IRS2), resulting in reduced activation of PI3K and AKT. Reduction in AKT phosphorylation decreases the glycogen synthase (GS) mediated glycogen synthesis and increases gluconeogenesis, which in turn leads to glucose release through GLUT2 [18]. Various studies in the literature report that the concentrations of both GLUT2 protein and mRNA were increased 1.6- to 2-fold in diabetic rat livers [21, 22]. PIP3 plays an important role in the insulin signaling pathway [3]. Cellular PIP3 concentration is regulated by PI3K/PTEN equilibrium, and upon formation, PIP3 causes the activation of AKT, leading to increased glucose metabolism. However, there are no previous reports in the literature concerning whether hepatic PIP3 levels are altered in diabetes.

Results from this in vivo study document that, while livers from T1D and T2D rats show a reduction in hepatic PIP3 levels, phosphorylation of AKT, and an increase in GLUT2 protein expression compared to those of controls, the PIP2 levels remain unchanged. This suggests that a decrease in hepatic levels of PIP3 but not those of PIP2, may be linked to downregulation of AKT phosphorylation. This can contribute to impaired glycogen synthesis and increased gluconeogenesis, and is associated with increased hepatic glucose output as manifested by upregulation of GLUT2 protein expression. This study demonstrates for the first time that a decrease in hepatic PIP3 levels may have a role in altered glucose homeostasis in diabetes.

Diabetes is associated with hyperglycemia and increased oxidative stress, which can upregulate PTEN and downregulate PI3K, leading to a decrease in cellular PIP3. This study demonstrates for the first time a decrease in hepatic levels of PIP3 but not those of PIP2, which may have a role in the downregulation of AKT/ GLUT2 protein expression and glucose metabolism in diabetes. Further studies are needed to investigate the direct effect of PIP3/PIP2 in hepatocytes to understand the causal relationship of PIP3/PIP2 with glucose metabolism. Future studies on the status of PIP3 in other tissues are needed to further evaluate the role of PIP3 as a biomarker for insulin resistance and impaired glucose homeostasis in diabetes. This could lead in turn to new therapeutics that target improvement of PIP3 status in diabetes.

\section{Conflict of interest statement}

The authors declare that they have no conflict of interest.

\section{Acknowledgements}

The authors are supported by grants from NIDDK and the Office of Dietary Supplements of the National Institutes of Health R01 DK072433 and the Malcolm Feist Endowed Chair in Diabetes. This study is also funded by a fellowship from the Malcolm Feist Cardiovascular Research Endowment, LSU health Sciences Center, Shreveport. The authors thank Ms Georgia Morgan for excellent editing of this manuscript 


\section{Cellular Physiology Cell Physiol Biochem 2012;30:1363-1370 \begin{tabular}{l|l|l}
\hline DOI: $10.1159 / 000343325$ & 02012 S. Karger AG, Basel
\end{tabular} \begin{tabular}{l|l} 
and Biochemistry Published online: October 30, 2012 & www.karger.com/cpb
\end{tabular}}

Manna/Jain: PIP3, PIP2 and Hepatic Glucose Homeostasis

\section{References}

1 Pendaries C, Tronchère H, Plantavid M, Payrastre B: Phosphoinositide signaling disorders in human diseases. FEBS Lett 2003;546:25-31.

2 Czech MP: PIP2 and PIP3: complex roles at the cell surface. Cell 2000;100:603-606.

- Hinchliffe KA: Cellular signalling: stressing the importance of PIP3. Curr Biol 2001;11:R371-R372.

-4 Park WS, Heo WD, Whalen JH, O’Rourke NA, Bryan HM, Meyer T, Teruel MN: Comprehensive identification of PIP3-regulated PH domains from C. elegans to H. sapiens by model prediction and live imaging. Mol Cell 2008;30:381-392.

5 Várnai P, Bondeva T, Tamás P, Tóth B, Buday L, Hunyady L, Balla T: Selective cellular effects of overexpressed pleckstrin-homology domains that recognize PtdIns(3,4,5)P3 suggest their interaction with protein binding partners. J Cell Sci 2005;118:4879-4888.

6 Lietzke SE, Bose S, Cronin T, Klarlund J, Chawla A, Czech MP, Lambright DG: Structural basis of 3-phosphoinositide recognition by pleckstrin homology domains. Mol Cell 2000;6:385-394.

7 Toker A The synthesis and cellular roles of phosphatidylinositol 4,5-bisphosphate. Curr Opin Cell Biol 1998;10:254-261.

8 Taniguchi CM, Tran TT, Kondo T, Luo J, Ueki K, Cantley LC, Kahn CR: Phosphoinositide 3-kinase regulatory subunit p85alpha suppresses insulin action via positive regulation of PTEN. Proc Natl Acad Sci USA 2006;103:12093-12097.

-9 Butler M, McKay RA, Popoff IJ, Gaarde WA, Witchell D, Murray SF, Dean NM, Bhanot S, Monia BP: Specific inhibition of PTEN expression reverses hyperglycemia in diabetic mice. Diabetes 2002;51:1028-1034.

10 Ono H, Katagiri H, Funaki M, Anai M, Inukai K, Fukushima Y, Sakoda H, Ogihara T, Onishi Y, Fujishiro M, Kikuchi M, Oka Y, Asano T: Regulation of phosphoinositide metabolism, AKT phosphorylation, and glucose transport by PTEN (Phosphatase and Tensin Homolog Deleted on Chromosome 10) in 3T3-L1 adipocytes. Mol Endocrinol 2001;15:1411-1422.

-11 Saltiel AR, Kahn CR: Insulin signalling and the regulation of glucose and lipid metabolism. Nature 2001;414:799-806

12 Manna P, Jain SK: Hydrogen sulfide and L-cysteine increase phosphatidylinositol 3,4,5-trisphosphate (PIP3) and glucose utilization by inhibiting phosphatase and tensin homolog (PTEN) protein and activating phosphoinositide 3-kinase (PI3K)/serine/threonine protein kinase (AKT)/protein kinase C $/ \lambda(\mathrm{PKC} \zeta / \lambda)$ in 3T3L1 adipocytes. J Biol Chem 2011; 286: 39848-39859.

13 Funaki M, DiFransico L, Janmey PA: PI-4,5-P2 stimulates glucose transport activity of GLUT4 in the plasma membrane of 3T3-L1 adipocytes. Biochim Biophys Acta 2006;1763:889-899.

14 Gray A, Olsson H, Batty IH, Priganica L, Peter Downes C: Nonradioactive methods for the assay of phosphoinositide 3-kinases and phosphoinositide phosphatases and selective detection of signaling lipids in cell and tissue extracts. Anal Biochem 2003;313:234-245.

15 Levinthal GN, Tavill AS: Liver disease and diabetes mellitus. Clin Diabetes 1999;17.

16 Leturque A, Brot-Laroche E, Le Gall M, Stolarczyk E, Tobin V: The role of GLUT2 in dietary sugar handling. J Physiol Biochem 2005,61:529-537.

17 Zhao FQ, Keating AF: Functional properties and genomics of glucose transporters. Curr Genomics 2007;8:113-128.

-18 Jornayvaz FR, Shulman GI: Diacylglycerol activation of protein kinase C $\varepsilon$ and hepatic insulin resistance. Cell Metab 2012;15:574-584.

-19 Hwang JH, Perseghin G, Rothman DL, Cline GW, Magnusson I, Petersen KF, Shulman GI: Impaired net hepatic glycogen synthesis in insulin-dependent diabetic subjects during mixed meal ingestion. A 13C nuclear magnetic resonance spectroscopy study. J Clin Invest 1995;95:783-787

20 Aiston S, Peak M, Agius L: Impaired glycogen synthesis in hepatocytes from Zucker fatty fa/fa rats: the role of increased phosphorylase activity. Diabetologia 2000;43:589-597.

-21 Slieker LJ, Sundell KL, Heath WF, Osborne HE, Bue J, Manetta J, Sportsman JR: Glucose transporter levels in tissues of spontaneously diabetic Zucker fa/fa rat (ZDF/drt) and viable yellow mouse (Avy/a). Diabetes 1992;41:187-193.

-22 Yamamoto T, Fukumoto H, Koh G, Yano H, Yasuda K, Masuda K, Ikeda H, Imura H, Seino Y: Liver and musclefat type glucose transporter gene expression in obese and diabetic rats. Biochem Biophys Res Commun 1991;175:995-1002. 